\title{
Development of a Capacitive Pressure Sensor Based on Havar Alloy
}

\section{Diaphragm}

\author{
Shoubin LIU ${ }^{1, a^{*}}$, Bingwu ZHANG ${ }^{2, b}$ \\ ${ }^{1,2}$ School of Mechanical Engineering and Automation, Harbin Institute of Technology Shenzhen \\ Graduate School, Shenzhen, 518055, P. R. China \\ amesbliu@hit.edu.cn, ${ }^{\mathrm{b}} \mathrm{zbw0915@163.com}$ \\ ${ }^{*}$ Corresponding author
}

\begin{abstract}
Keywords: Capacitive pressure sensor, Sensing diaphragm, Havar alloy, ANSYS Workbench
\end{abstract} Abstract. This paper proposes a new pressure sensor using Havar alloy film as the sensing diaphragm, which has higher strength and toughness, better corrosion resistance and magnetic resistance as compared to traditional films. 2D distribution graphs of stress and strain of the diaphragm under a given pressure are analyzed with ANSYS Workbench. The diaphragm sensitivity and nonlinear error can be calculated by constructing the curve of the displacement of diaphragm center varied with input pressures. Two key parameters, thickness and diameter of the diaphragm are solved by minimizing the nonlinear error of diaphragm. $\mathrm{C} / \mathrm{F}$ and $\mathrm{F} / \mathrm{V}$ conversion techniques are used in capacitance detection circuit for converting weak capacitive signal to voltage signal. A prototype sensor is constructed and the calibration experiment is conducted. Calibration data analyzing shows that the linearity of the pressure sensor is $0.03 \%$ in the working range of 0 to $35 \mathrm{kPa}$, which indicates that the proposed capacitive pressure sensor using Havar alloy diaphragm possesses high accuracy.

\section{Introduction}

Capacitive pressure sensors have high sensitivity and simple structure, which are slightly impacted by the change of temperature and have good dynamic response characteristics[1,2]. The typical structure of a capacitive pressure sensor is shown in Fig. 1. There is a differential capacitor in the sensor, which consists of three capacitor plates. Two capacitor plates are two fixed electrodes and fabricated by vacuum-sputtering two conductive layers on two concave surfaces of glass. The third capacitor plate is the thin film or sensing diaphragm, acting as a movable electrode. The left and right chambers of the sensing diaphragm are filled with silicone oil to carry out the external pressure transmitted to the diaphragm of the sensor. The working principle is that the displacement of the movable diaphragm center is linearly related to the external load (pressure or differential pressure), which results the capacitance value of the differential capacitor varying linearly with the external load. Therefore, the external load can be derived by detecting the capacitance value of the sensor [3, 4].

Sensing diaphragms are the core components in capacitive pressure sensors. The measuring characteristics of a sensor, such as sensitivity and linearity, are limited by the mechanical and chemical characteristics of the selected diaphragm material $[5,6]$. Traditional materials of sensing diaphragms include copper, stainless steel and other metallic materials. Havar alloy is a newly emerging constant elastic alloy from Co-Fe series materials. It has many advantages, such as good dynamic response, good temperature stability and good elasticity. The chemical composition of Havar alloy is shown in Table 1. This paper tries to develop a new capacitive pressure sensor using 
Havar alloy as the sensing diaphragm. Strain and stress analysis, mode analysis and parameter optimization of the Havar diaphragm are carried out. Circuit design and calibration experiment of a prototype sensor are then fulfilled as follows.

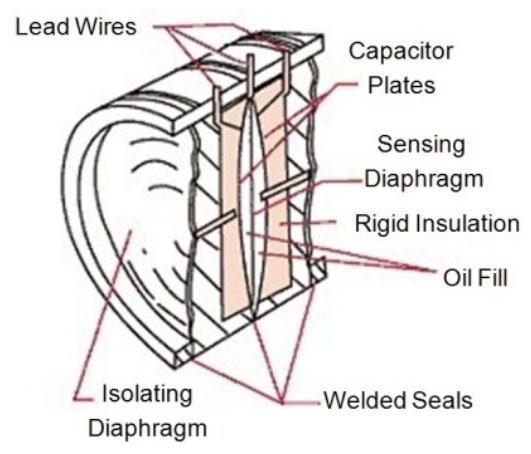

Fig. 1. Diagram of a capacitive pressure sensor

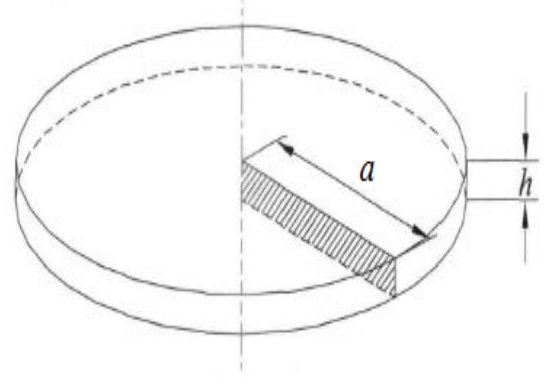

Fig. 2. Simplified model of sensing diaphragm

Table 1. Chemical composition of Havar alloy

\begin{tabular}{lcccccccc}
\hline Element & $\mathrm{Co}$ & $\mathrm{Cr}$ & $\mathrm{Ni}$ & $\mathrm{W}$ & $\mathrm{Mo}$ & $\mathrm{Mn}$ & $\mathrm{C}$ & $\mathrm{Fe}$ \\
\hline Percent(\%) & 42 & 19.5 & 12.7 & 2.7 & 2.2 & 1.6 & 0.2 & 19.1 \\
\hline
\end{tabular}

\section{Analysis and Optimization of the Havar Diaphragm}

Considering that the sealing characteristics and silicone oil of the sensor have no influence on the stress and strain analysis of the Havar diaphragm, the structure of Havar diaphragm is simplified as Fig.2. Thickness and diameter are two key parameters of the diaphragm. After numerical analysis of the diaphragm with ANSYS Workbench, two parameters of the diaphragm are optimized by minimizing the nonlinear error of the displacement of diaphragm center.

\section{Numerical Analysis of the Diaphragm}

In general engineering conditions, the thickness of diaphragm is usually not below $0.035 \mathrm{~mm}$, thus the initial thickness $h$ of the diaphragm can be set as $0.04 \mathrm{~mm}$. The initial diameter of the diaphragm is set as $32 \mathrm{~mm}$ (radius $a=16 \mathrm{~mm}$ ). By applying the input pressures of $0 \mathrm{kPa}, 7 \mathrm{kPa}, 14 \mathrm{kPa}$, $21 \mathrm{kPa}, 28 \mathrm{kPa}, 35 \mathrm{kPa}, 42 \mathrm{kPa}, 49 \mathrm{kPa}, 56 \mathrm{ka}$ and $63 \mathrm{kPa}$ to the $3 \mathrm{D}$ finite element model of Havar diaphragm, the ANSYS Workbench can perform stress and strain analysis. A set of results under $P=49 \mathrm{kPa}$ is shown in Fig. 3 and Fig.4. By collecting the data of maximum deflection values under different pressure points, we can generate the curve of the displacement of diaphragm center varied with input pressures, i.e. the elasticity curve of the diaphragm, as shown in Fig.5. A stainless steel diaphragm (3J53) with the same structure is analyzed at the same time. Based on elasticity curves shown in Fig.5, we can obtain that the sensitivity of Havar diaphragm is $3.5 \mu \mathrm{m} / \mathrm{kPa}$, while the sensitivity of $3 J 53$ diaphragm is $3.3 \mu \mathrm{m} / \mathrm{kPa}$. Both sensitivity and linearity of Havar diaphragm are better than that of $3 \mathrm{~J} 53$ diaphragm. Fig. 6 shows displacement of diaphragm in the radial path under a pressure point of $35 \mathrm{kPa}$. The displacement of Havar diaphragm is closer to the theoretical value, as compared to that of $3 \mathrm{~J} 53$ diaphragm. 


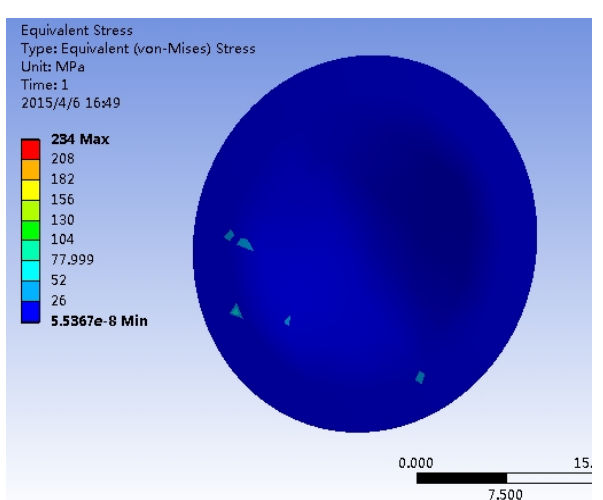

Fig. 3. Stress of Havar diaphragm under $P=49 \mathrm{kPa}$

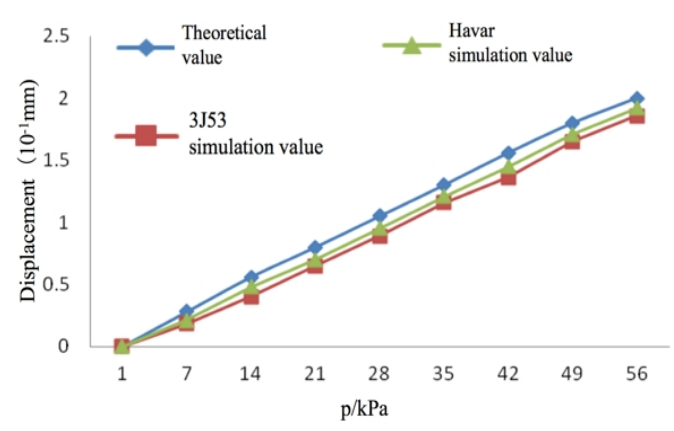

Fig.5. Displacement of diaphragm center under different $P$

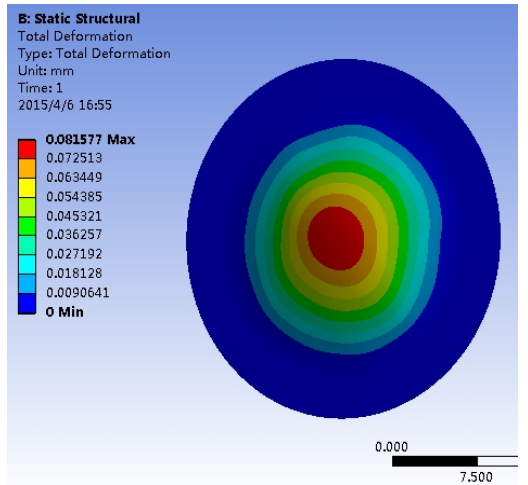

Fig. 4. Strain of Havar diaphragm under $P=49 \mathrm{kPa}$

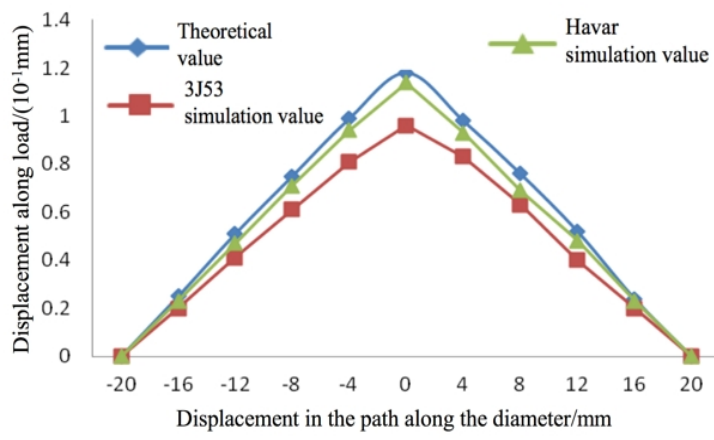

Fig.6. Displacement of diaphragm in the radial path under $P=35 \mathrm{kPa}$

Based on elasticity curves shown in Fig.5, we can also obtain the nonlinear error of the displacement of diaphragm center. As shown in Fig.7, it can be seen that the nonlinear error of the Havar diaphragm is better than that of $3 \mathrm{~J} 53$ diaphragm.

The diaphragm of a pressure sensor is a low-pass component, and its working frequency can be determined by the first-order natural frequency. Table 2 shows the results of mode analysis of the Havar diaphragm. The first-order natural frequency and vibration mode are shown in Fig.8. According to the first-order resonant frequency value of $8.964 \mathrm{kHz}$, we can set the general working bandwidth of the diaphragm is about $7.8 \mathrm{kHz}$.

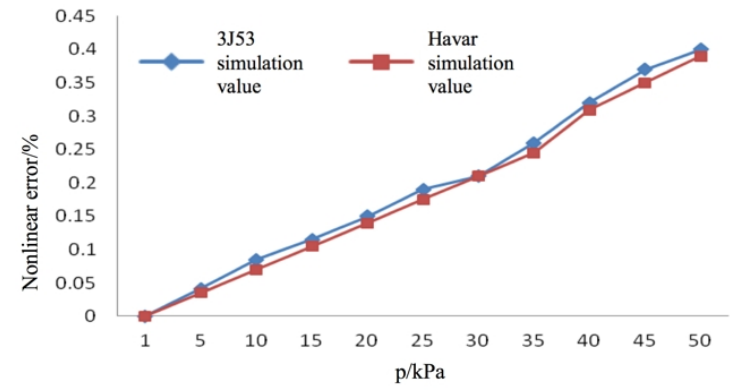

Fig. 7. Nonlinear error of the diaphragm

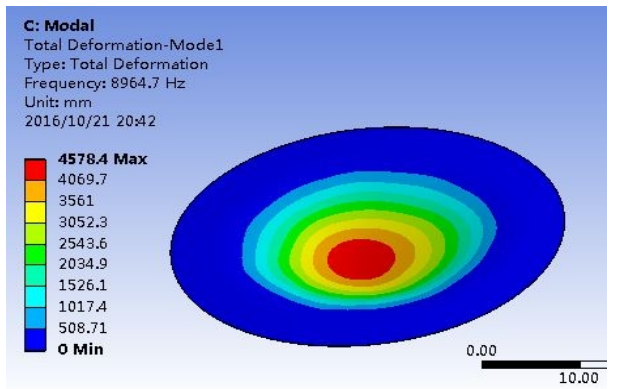

Fig. 8. $1^{\text {st }}$ natural frequency and vibration mode

Table 2. Vibration frequency values of the diaphragm under six modes

\begin{tabular}{lllllll}
\hline Mode & 1 & 2 & 3 & 4 & 5 & 6 \\
\hline Frequency $(\mathrm{Hz})$ & 8964.7 & 26476 & 27754 & 62735 & 66744 & 80135 \\
\hline
\end{tabular}

\section{Parameter Optimization of the Diaphragm}

It is assumed that the capacitive pressure sensor has a working pressure range of 0 to $35 \mathrm{kPa}$. In order to obtain optimized parameters of thickness and diameter of the diaphragm, by taking the 
radius $a$ and the thickness $h$ as the variables, an objective function is established to minimize the nonlinear error $f(x)$ of the diaphragm:

$$
\min _{x i_{\text {min }} \leq x i \leq x i_{\text {max }}} f(x)=(\Delta \varepsilon) Y_{F S}
$$

where $x i$ denotes the variable of radius $a$ or thickness $h$ of the diaphragm, $x i_{\text {min }}$ and $x i_{\text {max }}$ are the smallest and the largest design variables respectively, $\Delta \mathcal{E}$ are the nonlinear error, $Y_{F S}$ are the full scale of the sensor.

In addition, the radius $a$ and the thickness $h$ should be limited by the following constraints: (1) the $\sigma_{\max }$ (maximum equivalent stress) of the diaphragm should be less than [ $\left.\sigma\right]$ (allowable stress); (2) the maximum deflection $d_{H}$ of the diaphragm center should be less than spherical chord height $\left[\mathrm{d}_{0}\right]$; (3) the first order vibration frequency $f_{l}$ should be greater than [f] (natural frequency). The above constraints are summarized as:

$$
\left.\begin{array}{l}
\sigma_{\max } \leq[\sigma]=600 \mathrm{MPa} \\
d_{H} \leq\left[\mathrm{d}_{0}\right]=0.15 \mathrm{~mm} \\
f_{l} \geq[\mathrm{f}]=7800 \mathrm{~Hz}
\end{array}\right\}
$$

To solve above optimization problem, we first draw the $d_{H} \sim p$ curve (i.e., elasticity curve- the curve of the displacement of diaphragm center varied with $P$ ), with different film thickness under a fixed film radius $a=16 \mathrm{~mm}$, as shown in Fig.9. Input pressures are $0 \mathrm{kPa}, 7 \mathrm{kPa}, 14 \mathrm{kPa}, 21 \mathrm{kPa}, 28 \mathrm{kPa}$ and $35 \mathrm{kPa}$. It can be found that the sensitivity $S\left(S=d_{H} / P\right)$ decreases with the increase of film thickness $h$. Considering the factor of linearity, the selected film thickness is $h=5.0 \times 10^{-2} \mathrm{~mm}$. We then draw the $d_{H} \sim p$ curve with different film radius $a$ under a fixed film thickness $h=5.0 \times 10^{-2} \mathrm{~mm}$, as shown in Fig.10. It can be found that the sensitivity $S$ increases with the increase of film radius $a$. In order to obtain a minimized nonlinear error, the selected film radius is $a=13 \mathrm{~mm}$. Finally, we get the optimized structure parameters of Havar diaphragm, as listed in Table 3.

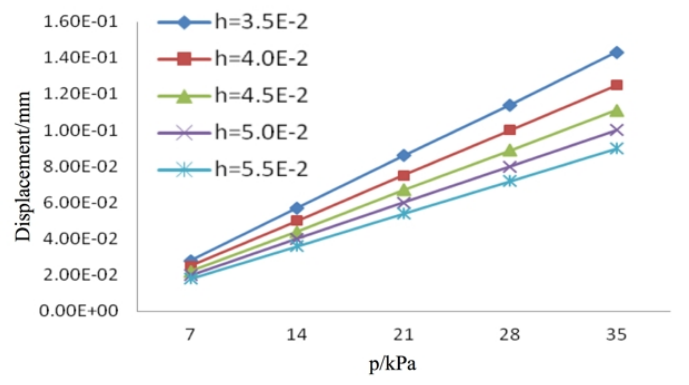

Fig. 9. Elasticity curve varied with film thickness under $a=16 \mathrm{~mm}$

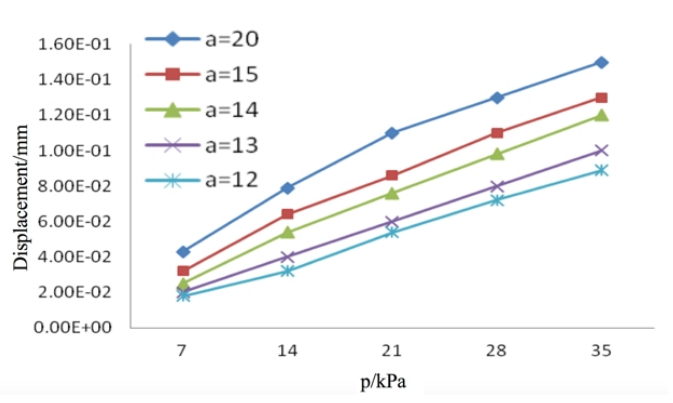

Fig. 10. Elasticity curve varied with film radius under $h=0.05 \mathrm{~mm}$

Table 3. Structure parameters of the Havar diaphragm

\begin{tabular}{lll}
\hline Item & Initial value & After optimization \\
\hline Radius $a(\mathrm{~mm})$ & 16 & 13 \\
Thickness $h(\mathrm{~mm})$ & 0.04 & 0.05 \\
Center displacement $d_{H}(\mathrm{~mm})$ & 0.05 & 0.08 \\
The first-order natural frequency $f_{l}(\mathrm{~Hz})$ & 8964 & 7100 \\
\hline
\end{tabular}




\section{Circuit Design}

The capacitance detection circuit is composed by power supply module, C/F conversion module, $\mathrm{F} / \mathrm{V}$ conversion module and voltage follower output module. The principle of capacitance detection is to covert the varied capacitance into a varied frequency signal. Such principle enables a wide range of capacitance measurement from a few $\mathrm{pF}$ to hundreds $\mathrm{pF}$. $\mathrm{C} / \mathrm{F}$ conversion module is the core of the sensor circuit. Fig. 11 shows the schematic diagram of the $\mathrm{C} / \mathrm{F}$ conversion circuit.

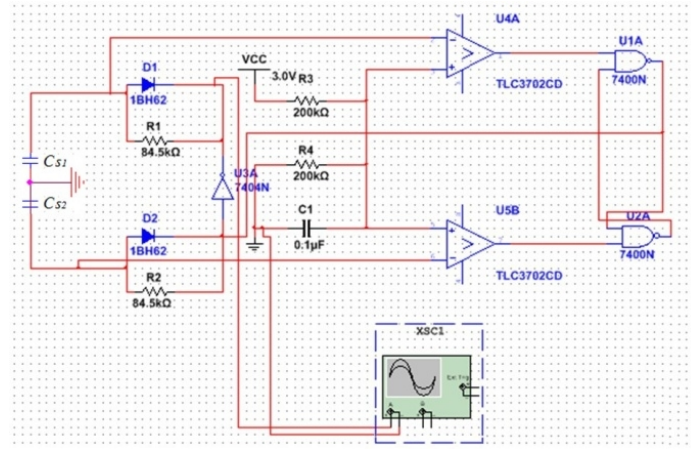

Fig. 11. Diagram of the $\mathrm{C} / \mathrm{F}$ conversion circuit

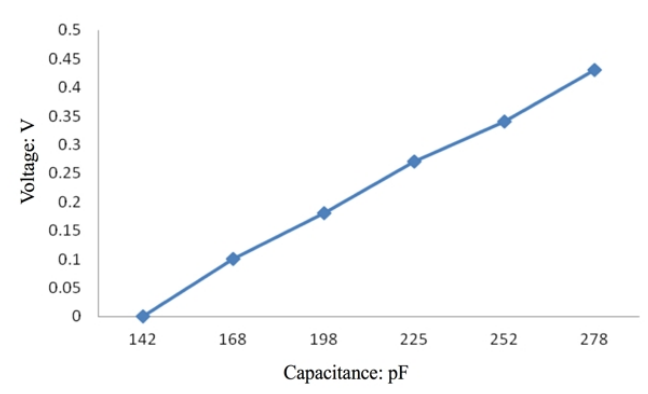

Fig. 13. Actual capacitance-voltage characteristic curve

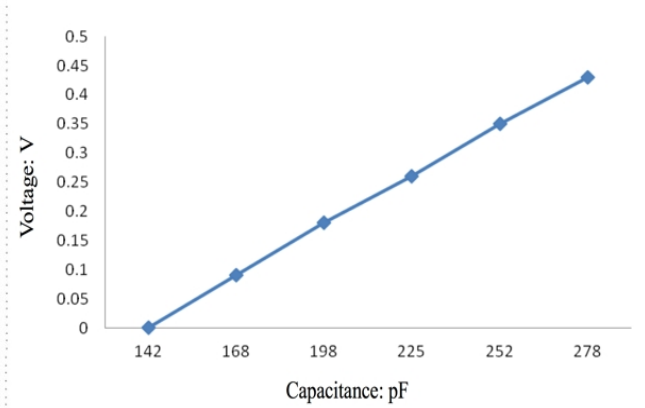

Fig. 12. Simulated capacitance-voltage curve

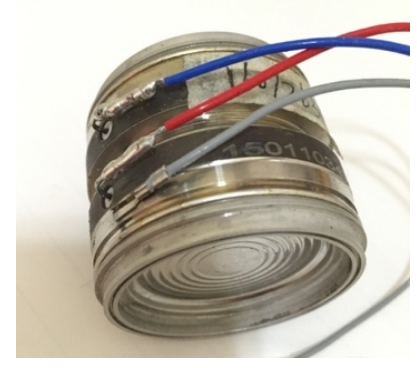

Fig. 14. The prototype sensor

A simulated capacitance-voltage $(C-V)$ curve of the capacitance detection circuit can be obtained by using the circuit simulation tool Multisim, as shown in Fig.12. It can be seen that capacitance as the input signal is strictly positively correlated with the output voltage. In order to test the actual circuit, the reference capacitance is set as $140 \mathrm{pF}$ while the circuit is respectively connected to capacitors of $168 \mathrm{pF}, 198 \mathrm{pF}, 225 \mathrm{pF}, 252 \mathrm{pF}$ and $278 \mathrm{pF}$. The actual $C-V$ characteristic curve is shown in Fig.13. The linearity of actual $C-V$ curve is little bit poor than that of simulated $C$ - $V$ curve. The distributed capacitance and parasitic capacitance in the actual PCB is further suppressed in manufacture of the prototype sensor.

\section{Calibration Experiment}

An engineering prototype sensor is fabricated, as shown in Fig.14. The capacitance detection circuit is installed inside the sensor. To test the overall measuring characteristics of the sensor, calibration experiment is carried out. The input pressure is increased from $0 \mathrm{kPa}$ to $35 \mathrm{kPa}$ by a step of $7 \mathrm{kPa}$ for the forward stroke, while the input pressure is reduced from $35 \mathrm{kPa}$ to $0 \mathrm{kPa}$ by a step of $7 \mathrm{kPa}$ for the reverse stroke. One forward stroke and one reverse stroke constitute a loop. We take three loops of testing, and obtain the calibration data shown in Table 4. Through least-squares data analyzing, we get measuring characteristics of the sensor as follows: Full-scale of output $Y_{F S}=407.928 \mathrm{mV}$; 
Linearity $\xi_{L}=0.030 \%$, Repeatability $\xi_{R}=0.048 \%$ and Hysteresis $\xi_{H}=0.040 \%$. These results indicate that the developed capacitive pressure sensor using Havar alloy diaphragm possesses high accuracy.

Table 4. Calibration data of the prototype sensor

\begin{tabular}{ccrrrrrr}
\hline Pressure (kPa) & & 0 & 7 & 14 & 21 & 28 & 35 \\
\hline & F1 & 0.261 & 81.840 & 163.450 & 245.108 & 326.665 & 408.09 \\
& R1 & 0.210 & 81.760 & 163.390 & 244.960 & 326.534 & \\
Output & F2 & 0.252 & 81.850 & 163.480 & 245.120 & 326.651 & 408.08 \\
Voltage (mV) & R2 & 0.204 & 81.788 & 163.370 & 244.960 & 326.551 & \\
& F3 & 0.259 & 82.830 & 163.500 & 245.114 & 326.672 & 408.12 \\
& R3 & 0.206 & 81.801 & 163.360 & 244.930 & 326.562 & \\
\hline
\end{tabular}

F-Forward stroke, $\mathrm{R}$ - Reverse stroke

\section{Conclusions}

This paper studies a new capacitive pressure sensor using Havar alloy diaphragm. ANSYS Workbench is used to analyze the mechanical properties of the Havar diaphragm, and the elasticity curve of the diaphragm under different conditions can be constructed. By minimizing the nonlinear error of the elasticity curve of diaphragm, two structure parameters, thickness and diameter of the diaphragm are determined. $\mathrm{C} / \mathrm{F}$ and $\mathrm{F} / \mathrm{V}$ conversion techniques are used in capacitance detection circuit for converting the weak capacitive signal to voltage signal. Calibration results shows that the linearity of the prototype sensor is $0.03 \%$ in a working range of 0 to $35 \mathrm{kPa}$, which indicates that the proposed capacitive pressure sensor using Havar alloy diaphragm possesses high accuracy.

\section{References}

[1] L. Rosengren, J. Soderkvist and L. Smith: Sensors \& Actuators Vol. A31(1992), p. 200.

[2] T. Nagata, H. Terabe, S. Kuwahara, et al: Sensors \& Actuators Vol. A34(1992), p. 173.

[3] F. Giulio, R. Kasper, L. Lasse, et al: Sensors Vol.10 (2010), p.9541.

[4] M. S. Zarnik, D. Belavic and S. Macek: Microelectronics International Vol. 28(2011), p.31.

[5] R. C. Luo: IEEE/ASME Transactions on Mechatronics Vol. 1(1996), p. 39.

[6] S. Kartmann, F. Koch, P. Koltay, et al: Sensors \& Actuators Vol. 247(2016), p. 656. 